\section{Robustness of Global Asymptotic Stability in Indirect Field-Oriented Control of Induction Motors}

\author{
R. Reginatto and A. S. Bazanella
}

\begin{abstract}
The influence of the rotor time constant mismatch on the global stability of induction motors under indirect field oriented control (IFOC) is analyzed. A test for global stability is provided, based on necessary and sufficient conditions for the existence of quadratic Lyapunov functions for IFOC drives. This test yields robustness margins with respect to rotor time constant mismatches. A typical example is presented to show that the robustness margins obtained are significantly less conservative than the ones obtained with previously proposed stability criteria.
\end{abstract}

Index Terms-Global asymptotic stability, indirect field-oriented control (IFOC), induction motors, robustness.

\section{INTRODUCTION}

The indirect field oriented control (IFOC) methodology is widely and successfully applied to meet high performance requirements with induction motor drives. The commissioning of an IFOC drive requires the knowledge of the rotor time constant, a parameter which can vary largely during the drive's operation. The consequent mismatch between the actual value and the commissioned value causes loss of field orientation, implying important performance and stability problems [6], [9]-[12]. Analysis of the robust stability of IFOC drives is therefore a major issue and as such has been pursued in [1], [3]-[5], [7], [13], and [14].

The existence of parameter ranges for which IFOC drives can present several equilibrium points has been proved in [7] and a complete characterization of the dependence of the equilibria on the rotor time constant mismatches has been given in [4]. Local stability properties of these equilibria have been investigated in [1], [4], [14] through bifurcation analysis; conditions for nonexistence of neither saddle-node nor Hopf bifurcations were provided. Guidelines for setting the speed or position controller in order to guarantee a certain local asymptotic stability margin with respect to rotor time constant mismatches for a practical load range were given [2].

A quadratic Lyapunov function for IFOC drives has been derived in [7], proving its robust global asymptotic stability. A generalization of this Lyapunov function has allowed to derive explicit formulae to conclude about robust global asymptotic stability in [4]. A passivity based analysis has been used in [8] to conclude about robust global asymptotic stability in the special case of zero load operation. In this note, we completely characterize quadratic Lyapunov functions for IFOC drives in speed or position control tasks, thus generalizing the aforementioned results. A procedure for determining guaranteed global asymptotic stability margins is presented which is significantly less conservative than the ones obtained with the previous results, and guidelines for setting the speed (or position) controller are derived from these calculations.

The note is organized as follows. Section II formulates the problem and provides the complete model for the induction motor with IFOC; the model is valid both for proportional-integral rotor speed regulation and for rotor position regulation through proportional-derivative control. The model is parameterized in the rotor time constant mismatch,

Manuscript received July 2, 2001; revised May 13, 2002. Recommended by Associate Editor Hua Wang.

The authors are with the Department of Electrical Engineering, Universidade Federal do Rio Grande do Sul, 90035-190 Porto Alegre, Brazil (e-mail: romeu@delet.ufrgs.br; bazanela@delet.ufrgs.br).

Digital Object Identifier 10.1109/TAC.2003.814264 which allows for the robustness analysis. In Section III, an explicit condition to conclude about global asymptotic stability is provided, which also yields a test for robustness of the global stability property regarding rotor time constant mismatches. This test is explored in Section IV to obtain robust global asymptotic stability margins in an example, showing that it is much less conservative than the ones previously presented [3], [7]. Finally, Section V provides a discussion on the results obtained.

\section{SYSTEM MODEL AND EQUILIBRIA}

\section{A. IFOC Modeling}

We consider the current fed induction motor model expressed in a reference frame rotating at synchronous speed. In terms of state variables, this model can be written as

$$
\begin{aligned}
\dot{x}_{1} & =-c_{1} x_{1}-u_{1} x_{2}+c_{2} u_{3} \\
\dot{x}_{2} & =-c_{1} x_{2}+u_{1} x_{1}+c_{2} u_{2} \\
\dot{w} & =-c_{3} w+c_{4}\left[c_{5}\left(x_{2} u_{3}-x_{1} u_{2}\right)-T_{m}\right]
\end{aligned}
$$

where $x_{1}$ and $x_{2}$ represent the $q$-axis and $d$-axis rotor fluxes, respectively, $w$ is the rotor speed, $u_{1}, u_{2}$ and $u_{3}$ stand for the inputs-the slipping frequency, the $d$-axis and $q$-axis stator current components, respectively; $T_{m}$ is the load torque, which is assumed constant, and the " $c$ " parameters are all positive. In particular, $c_{1}$ represents the inverse of the rotor time constant, which is a critical parameter for indirect field oriented control.

In speed regulation applications the IFOC strategy is usually applied along with a PI speed loop as described by the following equations [7], [12]:

$$
\begin{aligned}
& u_{1}=\hat{c}_{1} \frac{u_{3}}{u_{2}} \\
& u_{2}=u_{2}^{0} \\
& u_{3}=k_{p}\left(w_{\text {ref }}-w\right)+k_{i} \int_{0}^{t}\left(w_{\text {ref }}-w\right)(\zeta) d \zeta
\end{aligned}
$$

where $\hat{c}_{1}$ is an estimate for the inverse rotor time constant $c_{1}, k_{p}$ and $k_{i}$ are the gains of the PI speed controller, $w_{\text {ref }}$ is the constant reference velocity and $u_{2}^{0}$ is a constant which defines the flux level.

The knowledge of $c_{1}$ is a key issue in IFOC commissioning. If $\hat{c}_{1}=$ $c_{1}$, that is, if we have a perfect estimate of the rotor time constant, we say that the IFOC is tuned, otherwise, it is said to be detuned. Accordingly, we define

$$
\kappa \triangleq \frac{\hat{c}_{1}}{c_{1}}
$$

as the degree of tuning. It is clear that $\kappa>0$ and the IFOC is tuned if and only if $\kappa=1$.

We parameterize the closed-loop system (1)-(3) with the control (4)-(6) (see Fig. 1) in terms of the degree of tuning $\kappa$, yielding a fourthorder system that can be described as

$$
\begin{aligned}
& \dot{x}_{1}=-c_{1} x_{1}+c_{2} x_{4}-\frac{\kappa c_{1}}{u_{2}^{0}} x_{2} x_{4} \\
& \dot{x}_{2}=-c_{1} x_{2}+c_{2} u_{2}^{0}+\frac{\kappa c_{1}}{u_{2}^{0}} x_{1} x_{4} \\
& \dot{x}_{3}=-c_{3} x_{3}-c_{4}\left[c_{5}\left(x_{2} x_{4}-u_{2}^{0} x_{1}\right)-T_{e}\right] \\
& \dot{x}_{4}=k_{c} x_{3}-k_{p} c_{4}\left[c_{5}\left(x_{2} x_{4}-u_{2}^{0} x_{1}\right)-T_{e}\right]
\end{aligned}
$$

where we have defined the new state variables $x_{3} \triangleq w_{\text {ref }}-w$ and $x_{4} \triangleq u_{3}$ and the new parameters

$$
k_{c} \triangleq k_{i}-k_{p} c_{3} \quad T_{e} \triangleq T_{m}+\frac{c_{3}}{c_{4}} w_{\mathrm{ref}} .
$$




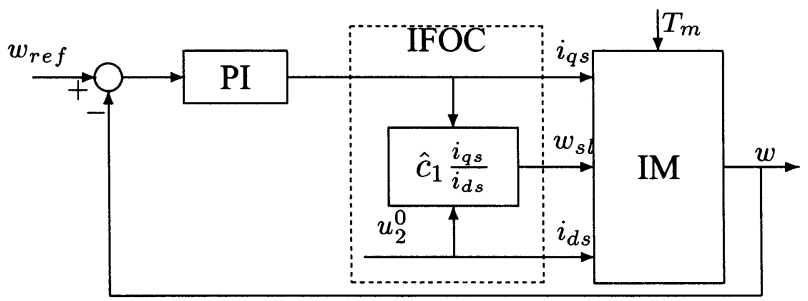

Fig. 1. Block diagram of IFOC.

For position regulation (6) is substituted by a proportional-derivative controller

$$
u_{3}=K_{p}\left(\delta_{\mathrm{ref}}-\delta\right)+K_{d} \frac{d\left(\delta_{\mathrm{ref}}-\delta\right)}{d t}
$$

where $\delta$ is the rotor position and $\delta_{\text {ref }}$ is the constant position reference. Defining the new state variables $x_{3}$ and $x_{4}$ as before (with $w_{\text {ref }}=0$ ) and observing that $\dot{\delta}_{\text {ref }}=0$ almost everywhere yields

$$
\begin{aligned}
\dot{x}_{4} & =-K_{p} \dot{\delta}-K_{d} \ddot{\delta}=K_{p} x_{3}-K_{d} \dot{w} \\
& =K_{c} x_{3}-K_{d} c_{4}\left[c_{5}\left(x_{2} x_{4}-x_{1} u_{2}^{0}\right)-T_{m}\right]
\end{aligned}
$$

which is the same as (11) but with $K_{c}=K_{p}-K_{d} c_{3}$ in lieu of $k_{c}$ and $K_{d}$ in lieu of $k_{p}$. Since the resulting dynamic model for the position regulation is the same as for speed regulation with zero reference speed, all the results derived for speed regulation are also valid for position regulation, and we henceforth treat only the constant speed regulation case.

\section{B. Tuned System}

A constant rotor flux must be established inside the motor before the systems can be operated. This is called the magnetization phase of IFOC, and is achieved by applying $u_{2}=u_{2}^{0}, u_{1}=0, w_{\text {ref }}=0$ to the motor in stand-still condition. The steady-state reached under these conditions is given by $x=x^{o}=\left[0,\left(c_{2} / c_{1}\right) u_{2}^{0}, 0,0\right]^{\prime}$, which is considered the initial state for IFOC operation.

In the case of tuned operation, $\kappa=1$, the model (8)-(11) simplifies considerably. First, notice that starting from $x(0)=x^{\circ}$, the fluxes $x_{1}$ and $x_{2}$ remain constant for all times, regardless of the behavior of $x_{4}$. Now, taking this into account, the remaining (10)-(11) can be rearranged as

$$
\begin{array}{r}
{\left[\begin{array}{l}
\dot{x}_{3} \\
\dot{x}_{4}
\end{array}\right]=\left[\begin{array}{cc}
-c_{3} & -\frac{c_{4} c_{5} c_{2} u_{2}^{0}}{c_{1}} \\
\left(k_{i}-k_{p} c_{3}\right) & -\frac{k_{p} c_{4} c_{5} c_{2} u_{2}^{0}}{c_{1}}
\end{array}\right]\left[\begin{array}{l}
x_{3} \\
x_{4}
\end{array}\right]} \\
+\left[\begin{array}{cc}
c_{3} & c_{4} \\
k_{p} c_{3} & k_{p}
\end{array}\right]\left[\begin{array}{c}
w_{\text {ref }} \\
T_{m}
\end{array}\right]
\end{array}
$$

which is a second-order linear system. We shall refer to the dynamic system (15) as the tuned system, which is usually taken as a base for setting the PI gains.

\section{Shifting the Equilibrium}

The equilibria of (8)-(11) are given by

$$
\left[\begin{array}{l}
x_{1}^{e} \\
x_{2}^{e} \\
x_{3}^{e} \\
x_{4}^{e}
\end{array}\right]=\left[\begin{array}{c}
\frac{c_{2} u_{2}^{0}}{c_{1}} \frac{1-\kappa}{1+\kappa^{2} r^{2}} r \\
\frac{c_{2} u_{2}^{0}}{c_{1}} \frac{1+\kappa r^{2}}{1+\kappa^{2} r^{2}} \\
0 \\
u_{2}^{0} r
\end{array}\right]
$$

where the dimensionless variables $r \triangleq x_{4}^{e} / u_{2}^{0}$ and $r^{*} \triangleq$ $\left(T_{e} c_{1}\right) /\left(c_{5} c_{2}\left(u_{2}^{0}\right)^{2}\right)$, have been defined. The constant $r^{*}$ represents the normalized load, since it is proportional to the electrical torque developed in steady state. The parameter $r$ can be shown to satisfy the polynomial equation [4]

$$
\kappa r^{3}-r^{*} \kappa^{2} r^{2}+\kappa r-r^{*}=0 .
$$

The equilibria are parameterized in terms of a single dimensionless quantity $r$, which satisfies (17). Each real solution of (17) represents an equilibrium. Since this is a third-order polynomial equation, there are either one or three equilibria. Since the coefficients of this equation are functions only of the degree of tuning $\kappa$ and the normalized load $r^{*}$, these two parameters completely define the equilibria. A complete characterization of equilibria has been given in [4] where it has been shown that the equilibrium is unique if and only if $\kappa \leq 3$.

Consider an arbitrary equilibrium and define the change of coordinates $z \triangleq x-x^{e}$. Writing the system (8)-(11) in these new coordinates and using (16) yields

$$
\dot{z}=\left[A_{0}\left(\kappa, r^{\star}\right)+z_{4} A_{1}(\kappa)\right] z
$$

with

$$
A_{0}\left(\kappa, r^{\star}\right)=\left[\begin{array}{cccc}
-c_{1} & -c_{1} r \kappa & 0 & c_{2}(1-\kappa) \frac{1+\kappa r^{2}}{1+k^{2} r^{2}} \\
r \kappa c_{1} & -c_{1} & 0 & \kappa c_{2} \frac{1-\kappa}{1+\kappa^{2} r^{2}} r \\
c_{4} c_{5} u_{2}^{0} & -c_{4} c_{5} u_{2}^{0} r & -c_{3} & -\frac{c_{4} c_{5} c_{2} u_{2}^{0}}{c_{1}} \frac{1+\kappa r^{2}}{1+\kappa^{2} r^{2}} \\
k_{p} c_{4} c_{5} u_{2}^{0} & -k_{p} c_{4} c_{5} u_{2}^{0} r & k_{c} & -\frac{k_{p} c_{4} c_{5} c_{2} u_{2}^{0}}{c_{1}} \frac{1+\kappa r^{2}}{1+\kappa^{2} r^{2}}
\end{array}\right]
$$

$$
A_{1}(\kappa)=\left[\begin{array}{cccc}
0 & -\frac{\kappa c_{1}}{u_{2}^{0}} & 0 & 0 \\
\frac{\kappa c_{1}}{u_{2}^{0}} & 0 & 0 & 0 \\
0 & -c_{4} c_{5} & 0 & 0 \\
0 & -k_{p} c_{4} c_{5} & 0 & 0
\end{array}\right]
$$

which presents an equilibrium at the origin $z=0$.

It can be seen from (16) and (17) that an operating condition is uniquely defined by the values of normalized load $r^{*}$ and degree of tuning $\kappa$. For any given operating condition, $A_{0}$ and $A_{1}$ are constant matrices, so that the system equation presents two terms: a linear term due to $A_{0}$ and a bilinear term given by $A_{1}$.

\section{MAIN RESULT}

Let us take a quadratic Lyapunov candidate

$$
V(z)=z^{T} P z, \quad P=P^{T}>0 .
$$

Then the derivative of $V$ along the trajectories of (18) is

$$
\begin{aligned}
\dot{V}(z)=z^{T}\left[A_{0}^{T}\left(\kappa, r^{*}\right) P+P A_{0}\left(\kappa, r^{*}\right)\right] z \\
+z_{4} z^{T}\left[A_{1}^{T}(\kappa) P+P A_{1}(\kappa)\right] z
\end{aligned}
$$

and we have the following result.

Theorem 1: Consider a given parameter mismatch $\kappa$ and normalized load $r^{*}$. If there exists a matrix $P$ satisfying the following conditions:

$$
\begin{aligned}
A_{1}^{T}(\kappa) P+P A_{1}(\kappa) & =0 \\
A_{0}^{T}\left(\kappa, r^{*}\right) P+P A_{0}\left(\kappa, r^{*}\right) & <0 \\
P=P^{T} & >0
\end{aligned}
$$

then the origin of (18) is globally asymptotically stable.

Proof: It is clear that (23) and (24) imply that the Lyapunov derivative (22) is negative definite globally and (25) implies that the Lyapunov candidate (21) is positive-definite globally.

Notice that these conditions are actually necessary and sufficient for a quadratic Lyapunov function to guarantee global asymptotic stability (g.a.s.).

Theorem 2: A quadratic Lyapunov function of the form (21) ensures global asymptotic stability of the origin of the system (18) for given 
parameter mismatch $\kappa$ and load $r^{*}$ if and only if there exists a matrix $P$ such that the matrix relations (24), (23), and (25) are satisfied.

Proof: The "if" part is given in the previous theorem. On the other hand, if (23) is violated there will always exist a $z_{4}$ which will make the second term in the derivative positive and larger in modulus than the first term, so that the derivative can not be globally negative in this case.

Conditions (24), (23) and (25) define a class of Lyapunov functions for the system (18) of quadratic form. Interestingly, any quadratic Lyapunov function must satisfy these conditions. Thus, in particular, the Lyapunov functions proposed in [3] and [7], which are also quadratic, are special cases belonging to this class. Indeed, the Lyapunov function proposed in [3] corresponds to a special choice of the $P$ matrix and satisfies $A_{1}^{T}(\kappa) P+P A_{1}(\kappa)=0$ for all $\kappa$.

These conditions are in the standard form of linear matrix inequalities and equalities (LMIs and LMEs, respectively) and as such can be solved with standard software. They provide a simple verification procedure to conclude about global asymptotic stability for any particular IFOC induction motor drive in any particular operating condition. They also provide a way to determine robust global asymptotic stability margins with respect to $\kappa$ for such systems, i.e., for a given PI setting and loading condition $r^{*}$ find, if possible, a range of $\kappa$ for which global asymptotic stability of system (18) is guaranteed. That such a range does exist around $\kappa=1$ for any PI setting and load condition has been proven elsewhere [3], [7]. Its determination can be accomplished through the following steps.

4) Define the range of interest for variation of the parameters $\mathcal{P} \triangleq$ $\left\{\left[\kappa_{\min }, \kappa_{\max }\right] \times\left[r_{\min }^{*}, r_{\max }^{*}\right]\right\}$ and a mesh of points inside this range.

5) For each point in the mesh above, run the LMI/LME problem (23), (24), and (25).

6) All the points in the mesh for which the problem is feasible represent a globally asymptotic stable operating condition.

\section{Determination of Robustness Margins}

The real solutions of (17) give the equilibrium values of $r$ for any given degree of tuning $-\kappa$ - and any given load $-r^{*}$. We know that (17) has a unique real solution for any load if and only if $\kappa \leq 3$ [4]. Hence, g.a.s. can be obtained for all load only within this range of parameter mismatch. This is also the practical range of variation of this parameter in most drives, as practical variations of the rotor time constant due to temperature and load variations are usually within $200 \%$ [9]. Accordingly, since in this note we are concerned with g.a.s., we consider only the parameter range $\kappa \times r^{*} \in\{(0,3) \times \Re\}$.

Theorem 1 provides a test for global stability which can be applied for any motor in any operating condition. This test is a generalization of the previously presented robustness criteria [4], [7], [8]. The matrix equations in the test depend on all the parameters of the drive, which can be divided into four sets

- the physical parameters of the motor (the ' $c$ ' parameters);

- the setting of the PI $\left(k_{p}\right.$ and $\left.k_{i}\right)$;

- the load $\left(r^{*}\right)$;

- the mismatch in the rotor time constant $(\kappa)$.

In order to get a better insight to the problem and establish typical robustness margins we apply the global stability test to data taken from a real induction motor. We aim to study, for a given motor and a given setting of the PI speed loop, what is the region of the parameter plane $\kappa \times r^{*}$ for which global stability is achieved. To this end, we apply the test with the $c$ parameters, $k_{p}$ and $k_{i}$ fixed, varying $\kappa$ and $r^{*}$ in the set of practical significance $\left(\kappa, r^{*}\right) \in(0,3) \times[0,2]$.

Furthermore, we perform this procedure for different settings of the PI speed loop, in order to verify its influence on the robustness of the

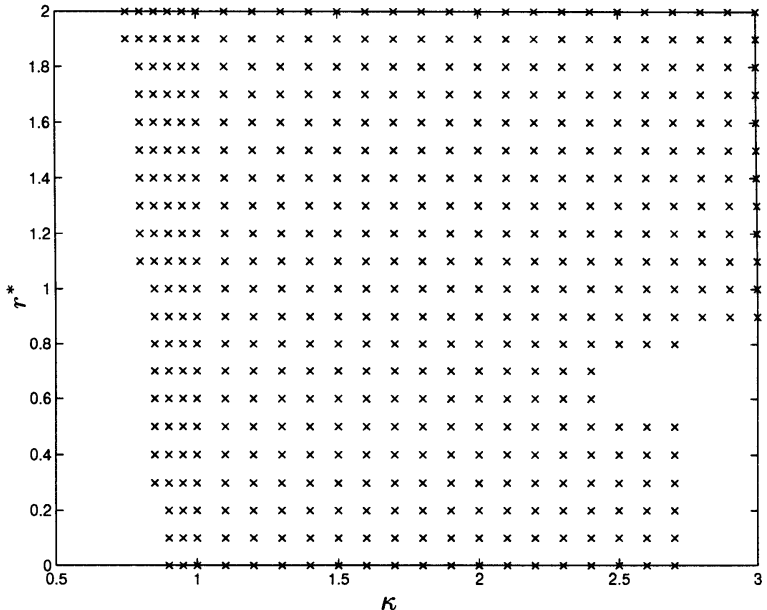

(a)

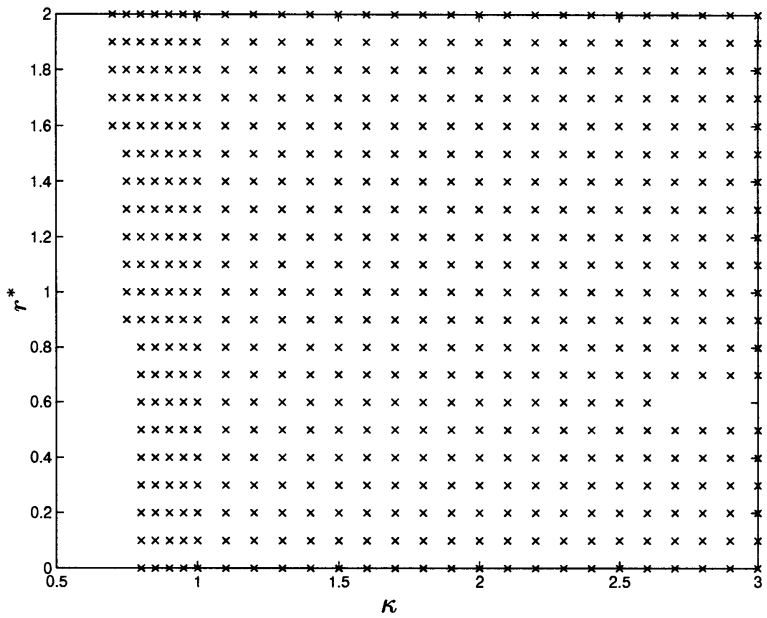

(b)

Fig. 2. Parameter range of g.a.s. for the 1-HP motor, $\eta=2$

global stability. The PI parameters are usually set in order to provide a desired performance to the system under the assumption of perfect tuning $(\kappa=1)$. The closed-loop poles of the tuned system are the roots of the characteristic polynomial

$$
p_{T}(s)=s^{2}+\left(c_{2}+k_{p} K\right) s+k_{i} K
$$

where $K \triangleq\left(c_{2} c_{4} c_{5} u_{2}^{0}\right) / c_{1}$, which can be arbitrarily assigned by choosing $k_{p}$ and $k_{i}$. We assume that the PI is set so that the tuned system's transient response is over damped and is $\eta$ times faster than the rotor time constant $c_{1}$, i.e,

$$
s^{2}+\left(c_{2}+k_{p} K\right) s+k_{i} K=\left(s+\eta c_{1}\right)^{2} .
$$

Then the parameter $\eta$ is used to represent the PI setting. That this is a one to one parameter mapping has been shown in [2]. It was also shown in [2] that the equilibrium point is locally asymptotically stable for all $\kappa \in(0,3)$ and $r^{*} \in[0,2]$ provided that $\eta \leq 23$.

Remark 1: Other choices of closed-loop poles in (27) could be considered for this analysis. For instance, $p_{T}(s)=s^{2}+2 \eta c_{1} s+2 \eta^{2} c_{1}^{2}$ would be a choice for a critically damped transient response and the above analysis could be easily replicated for this case. The choice (27) is motivated by the observation that the choice of real eigenvalues seems to be the most favorable one regarding the stability margins and transient performance [2].

In order to verify the effectiveness of the proposed global stability test (Theorem 1), we compare the region in the parameter space $\kappa \times$ $r^{*}$ for which global asymptotic stability of IFOC is guaranteed by the 

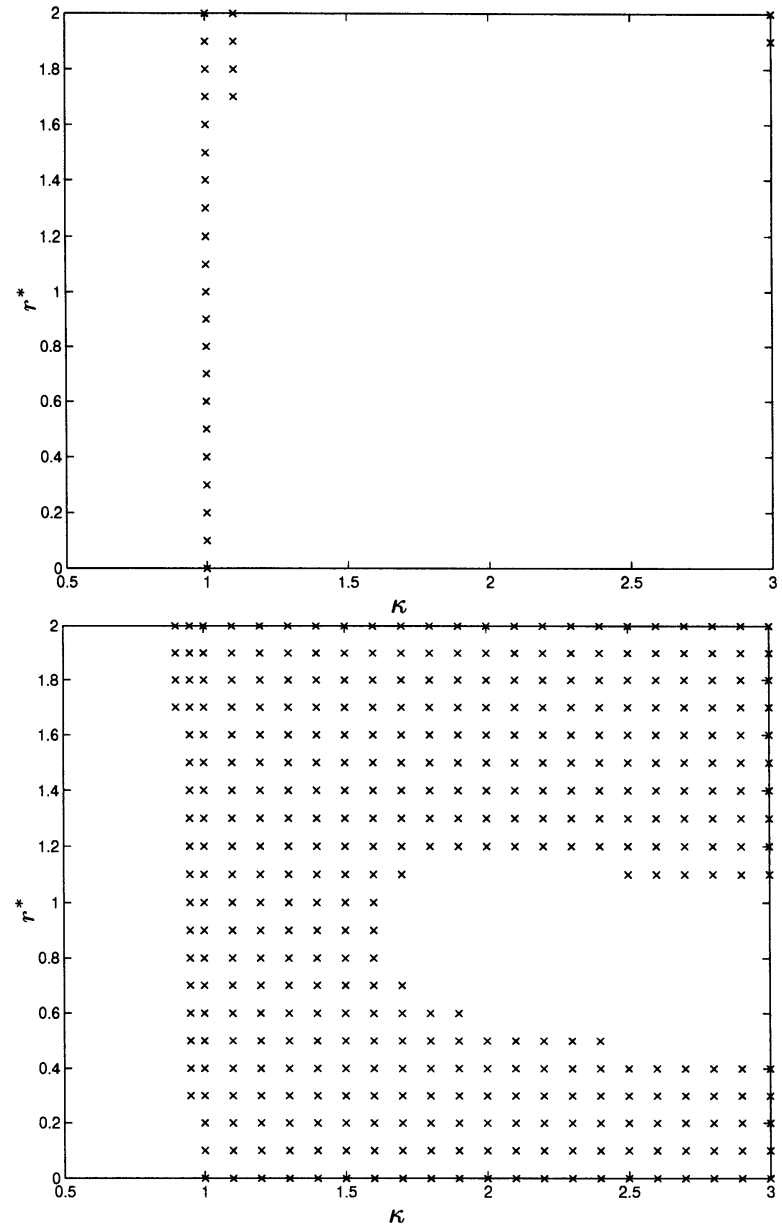

Fig. 3. Parameter range of g.a.s. for the 1-HP motor, $\eta=10$.

proposed test with the one guaranteed by the test given in [4]. Recall that the class of Lyapunov functions from which the test of [4] is derived is a subset of the class defined in this note. Recall also that the Lyapunov function given in [7] is a particular case in this subset.

We take data from a three phase induction motor, with 1-HP rated power output and $220-\mathrm{V}$ rated line voltage. The parameters of the motor are given in the Appendix. Let the parameters of the PI be chosen as in (27) with $\eta=2$. Then, we apply the global stability test for $\kappa$ and $r^{*}$ over a grid in the rectangle $(0,3) \times[0,2]$. Fig. 2 shows the region of the parameter space $\kappa \times r^{*}$ for which the test gives a positive answer: plot (b) shows the results obtained with the LMI/LME criterion in this note; the results obtained with the criterion in [4] are shown in plot (a). The operating point is guaranteed to be globally asymptotically stable for all load and parameter mismatch in the dotted region.

One can see that the range of parameters for which g.a.s. is guaranteed is larger with the proposed LMI/LME criterion. As faster response is assigned through the PI settings, the improvement on the stability margins is even more evident. This can be seen in Figs. 3 and 4, which show the cases for $\eta=10$ and $\eta=20$. For faster system's response the new LMI/LME is far less conservative.

For moderate values of $\eta$, that is, when the system is not made too fast by the PI settings, global asymptotic stability is guaranteed for most practical values of $\kappa$ and $r^{*}$. As the system is made faster, the range of parameter values for which g.a.s. is guaranteed gets smaller, particularly for $\kappa<1$. For $\eta=20$ this range is much smaller than the practical range of interest. Recall that for $\eta>23$ not even local stability is obtained for all $\kappa$ in the range $(0,3)$.
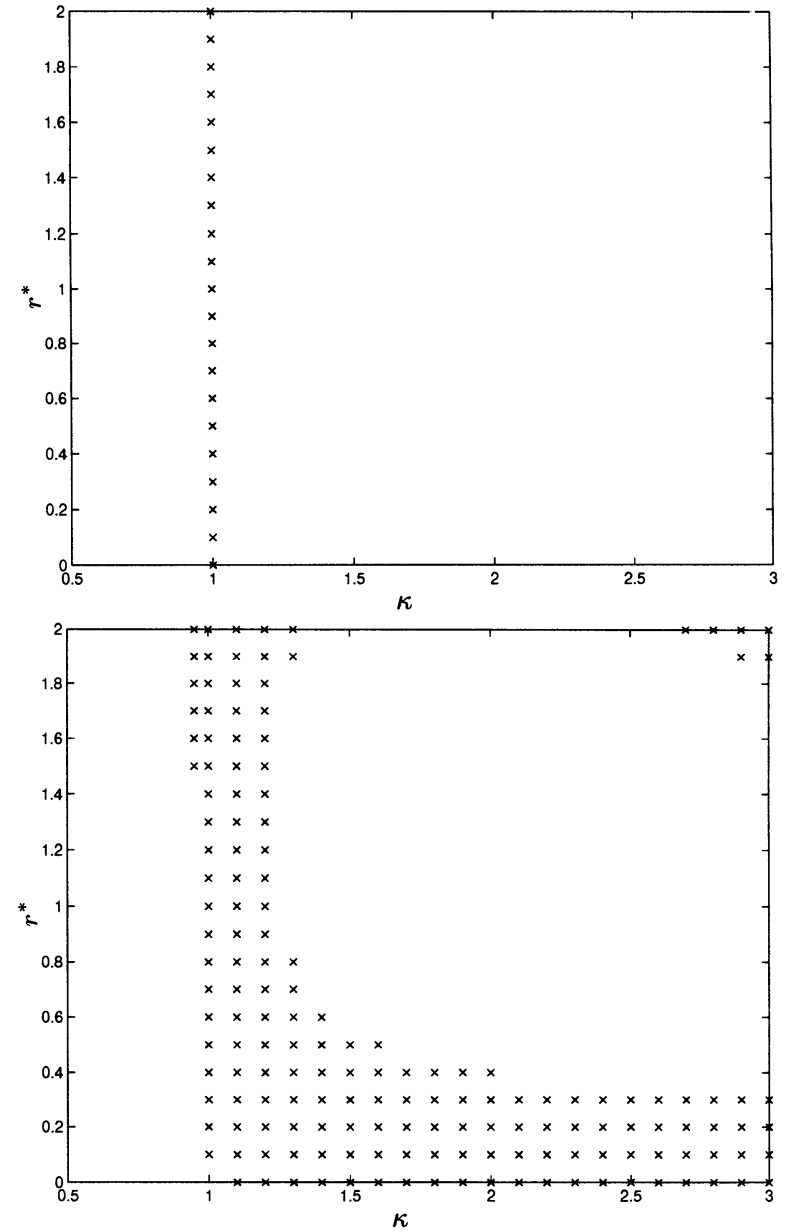

Fig. 4. Parameter range of g.a.s. for the $1-\mathrm{HP}$ motor, $\eta=20$.

\section{DISCUSSION}

We have provided a test for robust global asymptotic stability of IFOC which can be easily implemented provided the physical parameters of the motor are known. This test provides allowable margins of errors in the rotor time constant for any given IFOC drive.

The proposed test generalizes previous results based on quadratic Lyapunov functions. It provides the largest global asymptotic stability margins for IFOC, with respect to rotor time constant mismatches, that can be estimated by means of quadratic Lyapunov functions. Results provided for a particular IFOC drive have confirmed such improvement with respect to previous methods.

The proposed robust stability test also provides a means to tuning the PI speed/position regulators for IFOC drives. By choosing practical ranges of parameter and load variations ( $\kappa$ and $\left.r^{*}\right)$, global asymptotic stability can be ensured for that range by iteratively applying the stability test for different PI settings. Rules of thumb can also be derived from these results as guidelines for such tuning: the speed loop should not be made too fast, as the robustness margins would become too small. This rule is consistent with the local asymptotic stability analysis provided in [1], [3], [7], and [8].

\section{APPENDIX}

\begin{tabular}{|c|c|c|c|}
\hline$c_{1}$ & $13.7 \mathrm{~s}^{-1}$ & $c_{2}$ & $1.56 \Omega$ \\
\hline$c_{3}$ & $0.59 \mathrm{~s}^{-1}$ & $c_{4}$ & $1.18 \mathrm{~kg}^{-1} \mathrm{~m}^{-2}$ \\
\hline$c_{5}$ & 2.86 & $u_{2}^{0}$ & $4 \mathrm{~A}$ \\
\hline
\end{tabular}




\section{REFERENCES}

[1] A. Bazanella, R. Reginatto, and R. Valiati, "On Hopf bifurcations in indirect field oriented control of induction motors: designing a robust PI controller," presented at the 38th Conf. Decision Control, Phoenix, AZ, Dec. 1999.

[2] A. S. Bazanella and R. Reginatto, "Robust tuning of the speed loop in indirect field oriented control of induction motors," Automatica, vol. 37, no. 11, pp. 1811-1818, November 2001.

[3] — , "Robustness margins for indirect field-oriented control of induction motors," in Proc. 37th Conf. Decision Control, Tampa, FL, Dec. 1998, pp. 1001-1006.

[4] - "Robustness margins for indirect field oriented control of induction motors," IEEE Trans. Automat. Contr., vol. 45, pp. 1226-1231, June 2000.

[5] _ - "Robustness of global asymptotic stability in indirect field oriented control of induction motors," presented at the 39th Conf. Decision Control, Dec 2000.

[6] B. K. Bose, Power Electronics and AC Drives. Upper Saddle River, NJ: Prentice-Hall, 1987.

[7] P. A. S. De Wit, R. Ortega, and I. Mareels, "Indirect field-oriented control of induction motors is robustly globally stable," Automatica, vol. 32, no. 10 , pp. 1393-1402, 1996.

[8] G. Espinosa-Perez, G. Chang, R. Ortega, and E. Mendes, "On field-oriented control of induction motors: tuning of the PI gains for performance enhancement," in Proc. Conf. Decision Control, Tampa, FL, Dec. 1998, p. WMI5-2.

[9] R. Krishnan and F. C. Doran, "Study of parameter sensitivity in highperformance inverter-fed induction motor drive systems," IEEE Trans. Ind. Applicat., vol. IA-23, pp. 623-635, Jul/Aug. 1987.

[10] W. Leonhard, Control of Electrical Drives. Berlin, Germany: Springer-Verlag, 1985.

[11] R. Marino, S. Peresada, and P. Valigi, "Adaptive input-output linearizing control of induction motor," IEEE Trans. Automat. Contr., vol. 38, pp. 208-221, Feb. 1993.

[12] D. W. Novotny and R. D. Lorenz, Introduction to Field Orientation and High Performance AC Drives. Piscataway, NJ: IEEE Press, 1986.

[13] R. Ortega, C. Canudas, and S. I. Seleme, "Nonlinear control of induction motors: torque tracking with unknown load disturbance," IEEE Trans. Automat. Contr., vol. 38, pp. 1675-1680, Nov. 1993.

[14] R. Ortega, P. J. Nicklasson, and G. E. Péres, "On speed control of induction motors," Automatica, vol. 32, no. 3, pp. 455-460, 1996

\section{Universal Disturbance Rejection for Nonlinear Systems in Output Feedback Form}

\author{
Zhengtao Ding
}

\begin{abstract}
This note deals with global disturbance rejection via output feedback of a class of uncertain nonlinear systems subject to a class of unknown disturbances. Both the uncertainty in the system model and the uncertainty in the exosystem are tackled concurrently. The disturbances generated from an unknown linear exosystem are completely rejected. The order of the exosystem is assumed known, and the eigenvalues are distinct. The system is assumed in the format of the minimum-phase output feedback form, with no knowledge of the values of any system parameters, including the high-frequency gain. No other assumptions are needed in the control design. A new set of filters are introduced for state estimation. The stability of the internal model is exploited to design a new auxiliary error, involving both the unknown parameters of the reformatted exosystem and those of the system, which makes it possible to group all the unknown parameters in a format suitable to adaptive control design. A Nussbaum gain is introduced in adaptive control design to tackle the unknown high-frequency gain and a number of control coefficients are also made adaptive so that the disturbance rejection is global with respect to unknown frequencies in the disturbances.
\end{abstract}

Index Terms-Adaptive control, backstepping, disturbances rejection, nonlinear systems, output regulation, uncertainty.

\section{INTRODUCTION}

One of the important concepts for disturbance rejection is the internal model principle. Recently, the internal model principle has been extensively exploited in the context of output regulation for nonlinear systems. In the seminal results shown in [1] and [2], the necessary and sufficient conditions for the existence of a local full information regulator are that the linearized system is stabilizable and there exists a certain invariant manifold [1]. A semiglobal extension to these results for a class of feedback linearizable systems is reported in [3] using a saturated high-gain observer [4]. Output regulation by error feedback is solved [5], [6] with the application of system immersion technique. A semiglobal adaptive output feedback control is presented in [7] for nonlinear systems represented by input-output models, using a high-gain observer. Global solutions for output regulation using state or partial-state feedback are shown for strict feedback systems in [8] and for extended strict feedback systems in [9]. Recently, a global result of robust regulation has been achieved for a class of nonlinear output feedback systems that cannot be linearized [10]. For the same class of nonlinear systems considered in [10], but with unknown parameters, an adaptive version of global output regulation has been achieved using a new dynamic swapping technique for state estimation in [11], and the result is extended to the case where the sign of high-frequency gain is unknown as well in [12].

A breakthrough in rejecting disturbances generated by an unknown exosystem is reported in [13], where an adaptive internal model is used to generate the parallel forward control component, based on a new formulation of the exosystem, for the complete rejection of disturbances with unknown frequencies for parametric-strict-feedback systems using state feedback. The new formulation of exosystem is soon used in semiglobal output regulation for nonlinear systems in normal

Manuscript received April 11, 2002; revised October 29, 2002 and January 21, 2003. Recommended by Associate Editor P. Tomei.

The author is with the Mechanical Engineering Division, School of Engineering, Ngee Ann Polytechnic, Singapore 599489, Republic of Singapore (e-mail: dzt@np.eud.sg).

Digital Object Identifier 10.1109/TAC.2003.814265 